\title{
Enhanced Oxygen Utilization Efficiency With Concomitant Activation of AMPK-TBC1D1 Signaling Nexus in Cyclophilin-D Conditional Knockout Mice
}

\section{OPEN ACCESS}

Edited by:

Giuseppe D'Antona,

University of Pavia, Italy

Reviewed by:

Shunchang Li,

Chengdu Sport University, China

Murugesan Rajaram,

The Ohio State University,

United States

Sakthivel Sadayappan,

University of Cincinnati,

United States

Ashutosh Kumar,

All India Institute of Medical Sciences

(Patna), India

*Correspondence: Jeejabai Radhakrishnan jeejabai.radhakrishnan@

rosalindfranklin.edu

Raúl J. Gazmuri

raul.gazmuri@rosalindfranklin.edu

Specialty section: This article was submitted to

Exercise Physiology,

a section of the journal

Frontiers in Physiology

Received: 10 August 2021 Accepted: 12 November 2021

Published: 08 December 2021

Citation:

Radhakrishnan J, Baetiong A and Gazmuri RJ (2021) Enhanced Oxygen

Utilization Efficiency With

Concomitant Activation of

AMPK-TBC1D1 Signaling Nexus in

Cyclophilin-D Conditional Knockout Mice.

Front. Physiol. 12:756659.

doi: 10.3389/fphys.2021.756659

\author{
Jeejabai Radhakrishnan ${ }^{1,2 *}$, Alvin Baetiong ${ }^{1}$ and Raúl J. Gazmuri ${ }^{1,2,3 *}$ \\ ${ }^{1}$ Resuscitation Institute, Rosalind Franklin University of Medicine and Science, North Chicago, IL, United States, \\ ${ }^{2}$ Department of Clinical Sciences, Rosalind Franklin University of Medicine and Science, North Chicago, IL, United States, \\ ${ }^{3}$ Captain James A. Lovell Federal Health Care Center, North Chicago, IL, United States
}

We have previously reported in HEK 293T cells and in constitutive cyclophilin-D (Cyp-D) knockout $(\mathrm{KO})$ mice that Cyp-D ablation downregulates oxygen consumption $\left(\mathrm{VO}_{2}\right)$ and triggers an adaptive response that manifest in higher exercise endurance with less $\mathrm{VO}_{2}$. This adaptive response involves a metabolic switch toward preferential utilization of glucose via AMPK-TBC1D1 signaling nexus. We now investigated whether a similar response could be triggered in mice after acute ablation of Cyp-D using tamoxifen-induced ROSA26Cre-mediated (i.e., conditional $\mathrm{KO}, \mathrm{CKO}$ ) by subjecting them to treadmill exercise involving five running sessions. At their first treadmill running session, $\mathrm{CKO}$ mice and controls had comparable $\mathrm{VO}_{2}\left(208.4 \pm 17.9\right.$ vs. $\left.209.1 \pm 16.8 \mathrm{ml} / \mathrm{kg} \mathrm{min}^{-1}\right), \mathrm{VCO}_{2}(183.6 \pm 17.2$ vs. $\left.184.8 \pm 16.9 \mathrm{ml} / \mathrm{kg} \mathrm{min}^{-1}\right)$, and RER $(0.88 \pm 0.043$ vs. $0.88 \pm 0.042)$. With subsequent sessions, $\mathrm{CKO}$ mice displayed more prominent reduction in $\mathrm{VO}_{2}$ (genotype \& session interaction $p=0.000$ ) with less prominent reduction in $\mathrm{VCO}_{2}$ resulting in significantly increased RER (genotype and session interaction $p=0.013$ ). The increase in RER was consistent with preferential utilization of glucose as respiratory substrate $(4.6 \pm 0.8$ vs. $4.0 \pm 0.9 \mathrm{mg} / \mathrm{min}, p=0.003)$. CKO mice also performed a significantly higher treadmill work for given $\mathrm{VO}_{2}$ expressed as a power $\mathrm{NO}_{2}$ ratio $\left(7.4 \pm 0.2 \times 10^{-3}\right.$ vs. $6.7 \pm 0.210^{-3}$ ratio, $p=0.025)$. Analysis of CKO skeletal muscle tissue after completion of five treadmill running sessions showed enhanced AMPK activation $(0.669 \pm 0.06$ vs. $0.409 \pm 0.11$ pAMPK $/ \beta$-tubulin ratio, $p=0.005)$ and TBC1D1 inactivation ( $0.877 \pm 0.16$ vs. $0.565 \pm 0.09$ pTBC1D1/ $\beta$-tubulin ratio, $p<0.05)$ accompanied by increased glucose transporter-4 levels consistent with activation of the AMPK-TBC1D1 signaling nexus enabling increased glucose utilization. Taken together, our study demonstrates that like constitutive Cyp-D ablation, acute Cyp-D ablation also induces a state of increased $\mathrm{O}_{2}$ utilization efficiency, paving the way for exploring the use of pharmacological approach to elicit the same response, which could be beneficial under $\mathrm{O}_{2}$ limiting conditions.

Keywords: oxygen consumption, conditional knockout, cyclophilin-D, exercise capacity, treadmill exercise 


\section{INTRODUCTION}

Cyclophilin-D (Cyp-D) is a mitochondrial matrix-resident peptidyl-prolyl isomerase involved in various pleotropic functions of the cell including energy metabolism, metabolic adaptation, and nuclear/mitochondria signaling, and recently in nuclear translocation of apoptosis inducing factor (Elrod et al., 2010; Tavecchio et al., 2013; Tubbs et al., 2014; Radhakrishnan et al., 2015, 2019; Qamar et al., 2021). We first reported in HEK $293 \mathrm{~T}$ cells that Cyp-D interacts with mitochondrial transcription factors and that Cyp-D silencing downregulates the expression of mitochondrial genes initiated from the heavy strand promoter 2 (HSP2) encoding respiratory complex proteins leading to a reduction in oxygen consumption $\left(\mathrm{VO}_{2}\right.$; Radhakrishnan et al., 2015). Further work in our laboratory showed that Cyp-D ablation using constitutive Cyp-D knockout (KO) mice also downregulated respiratory complex I and IV activities and thereby $\mathrm{VO}_{2}$ (Radhakrishnan et al., 2015). The $\mathrm{VO}_{2}$ downregulation was accompanied by a reduction in $\mathrm{VCO}_{2}$ but to a lesser extent resulting in an increased respiratory exchange ratio (RER) consistent with a metabolic shift favoring glucose utilization over fat. This effect was accompanied by enhanced exercise capacity demonstrating increased oxygen utilization efficiency (Radhakrishnan et al., 2019). The response was mediated in part via adenosine monophosphate-activated protein kinase (AMPK) and its downstream partner tre-2/USP6, BUB2, cdc16 domain family member 1 (TBC1D1), the so-called "AMPK-TBC1D1 signaling nexus."

We now investigated whether a similar adaptive response could be triggered in animals developed with normal Cyp-D expression after acutely inducing the Cyp-D ablation. To address this question, we used a conditional KO (CKO) mouse tamoxifen-induced ROSA26-Cre-mediated - and asked whether the acute Cyp-D ablation in CKO mice also: (i) reduces $\mathrm{VO}_{2}$ and improves exercise capacity, (ii) elicits a metabolic shift favoring glucose utilization, and (iii) whether AMPK-TBC1D1 signaling is involved. If the metabolic shift could be induced in adult mice born without the defect, the possibility of pharmacological manipulation became attractive, specially using small molecules to selectively destabilize the interaction between Cyp-D and mitochondrial transcription factors without necessarily affecting other Cyp-D functions and be beneficial for oxygen limiting clinical conditions.

\section{MATERIALS AND METHODS}

The studies were approved by the Institutional Animal Care and Use Committee at Rosalind Franklin University of Medicine and Science (Protocol B17-20) and by the Edward Hines VA Hospital Institutional Animal Care and Use Committee (Protocol H17-014).

\section{Animals}

Mice were purchased from the Jackson Laboratory (Bar Harbor, $\mathrm{ME}$, United States) and conditional Cyp-D KO mice were generated as described below in the Methods section. Mice were bred and group-housed in the Biologic Resource Facility (accredited by the Association for Assessment and Accreditation of Laboratory Animal Care) at the Rosalind Franklin University of Medicine and Science. Lights were set at the recommended illumination levels with a 12/12-h cycle controlled via automatic timers and the temperature maintained between 68 and $74^{\circ} \mathrm{F}$. Mice were fed high-quality commercial laboratory diets ad libitum.

\section{Materials}

NativePAGE Novex Bis-Tris gels (3-12\%), NativePAGE running buffer, NuPAGE Bis-Tris gels (4-12\%), NuPAGE MOPS SDS running buffer, NuPAGE transfer buffer, PVDF membrane, and SuperSignal West Femto maximum sensitivity substrate were obtained from Thermo Fisher Scientific Inc. (Brookfield, WI, United States). The antibodies phospho-AMPK (Thr 172), phospho-TBC1D1 (Ser 660), and $\beta$-tubulin were from Cell Signaling Technology (Danvers, MA, USA). Antibody for peroxisome proliferator activator receptor- $\gamma$ coactivator $1 \alpha$ (PGC-1 $\alpha$ ) was from Santa Cruz Biotechnology (Santa Cruz, CA, United States). All other fine chemicals were obtained from Sigma-Aldrich (St. Louis, MO, United States).

\section{Study Design}

Experiments were conducted in intact animals to assess the effects of conditional Cyp- $\mathrm{D}$ ablation in vivo on oxygen metabolism by measuring $\mathrm{VO}_{2}$ and derived parameters at rest and during exercise. Two groups of mice, age and gender matched, representing control $(n=10)$ and conditional Cyp-D KO $(n=10)$, were subjected to a treadmill running protocol until exhaustion, during which $\mathrm{VO}_{2}$, carbon dioxide production $\left(\mathrm{VCO}_{2}\right)$, and RER, total running time before exhaustion, total running distance, and work performed were measured. Fuel utilization was calculated using $\mathrm{VO}_{2}$ and $\mathrm{VCO}_{2}$ data. Five running sessions were performed. After the running sessions, mice skeletal muscle tissues were harvested, quickly frozen in liquid $\mathrm{N}_{2}$, and stored at $-80^{\circ} \mathrm{C}$ for Western blotting.

\section{METHODS AND MEASUREMENTS}

\section{Generation of Conditional Ppif Knockout Mice}

The Jackson Lab has cryopreserved sperm (stock 005737, Ppiftm1Mmos/J) that was heterozygous for the Cyp-D floxed allele $\left(\mathrm{Ppif}^{\mathrm{F} / /+}\right)$. This stock was originally generated by Dr. Stanley J. Korsmeyer's lab (Schinzel et al., 2005). Jackson Lab performed a cryorecovery from the frozen sperm using wildtype female (stock 000664, C57BL/6J) as oocyte donors. A heterogeneous Ppif colony $\left(\mathrm{Ppif}^{\mathrm{F} / /+}\right.$ ) was generated by the Jackson Lab.

Ppif $^{\mathrm{Pl} /+}$ mice from two different donating mothers were inter-crossed to generate homozygous Cyp-D floxed mice (Ppif ${ }^{\mathrm{Fl}}$ $\left.{ }^{\mathrm{Fl}}\right)$. Then, Ppif ${ }^{\mathrm{I} / \mathrm{Fl}}$ mice were crossed with mice homozygous for the ROSA26-Cre ${ }^{\mathrm{ERT} 2}$ (Cre/Cre) transgene (stock 008463; 
Gt(ROSA)26Sortm1(cre/ERT2)Tyj) to generate mice heterozygous for the Cyp-D floxed allele and hemizygous for ROSA26-Cre ${ }^{\text {ERT2 }}$ transgene $\left(\mathrm{Ppif}^{\mathrm{Fl} /+}-\mathrm{Cre} /{ }^{+}\right)$. Next, the $\mathrm{Ppif}^{\mathrm{HI} /+}-\mathrm{Cre}^{+}$mice were crossed with $\mathrm{Ppif}^{\mathrm{F} / \mathrm{Fl}}$ mice to generate conditional Cyp-D knockout mice $\left(\mathrm{Ppif}^{\mathrm{F} / / \mathrm{Fl}}-\mathrm{Cre}^{+}\right)$and controls $\left(\mathrm{Ppif}{ }^{\mathrm{F} / / \mathrm{Fl}}\right)$.

\section{Genotyping}

Genotyping was performed according to the recommendations by the Jackson Lab. Briefly, tail biopsy $(\sim 2 \mathrm{~mm})$ from 21-dayold pups were digested overnight at $55^{\circ} \mathrm{C}$ using $0.5 \mathrm{ml} /$ sample of DNA isolation buffer (100 mM Tris.Cl, pH 8, $5 \mathrm{mM}$ EDTA, $0.2 \%$ SDS, and $200 \mathrm{mM} \mathrm{NaCl}$ ) containing $5 \mu \mathrm{l}$ of $20 \mathrm{mg} / \mathrm{ml}$ proteinase $\mathrm{K}$. The samples were then heated at $95^{\circ} \mathrm{C}$ for $10 \mathrm{~min}$ to inactivate Proteinase K. Equal volume of phenol: $\mathrm{CHCl}_{3}$ : IAA mixture was then added, mixed gently for $5 \mathrm{~min}$ to allow precipitation of proteins, and centrifuged at $12,000 \mathrm{~g}$ for $5 \mathrm{~min}$ at $4^{\circ} \mathrm{C}$ for phase separation. The supernatant was carefully removed, 2 volumes of $100 \%$ ethanol were added, incubated at room temperature for $5 \mathrm{~min}$, and then centrifuged at $12,000 \mathrm{~g}$ for $5 \mathrm{~min}$ at $4^{\circ} \mathrm{C}$ for DNA precipitation. The supernatant was discarded, and the pellet (DNA) was washed with $1 \mathrm{ml}$ of $70 \%$ ethanol. The pellet was then dried at $37^{\circ} \mathrm{C}$ for $5 \mathrm{~min}$ and dissolved in TE buffer. The DNA concentration was determined by absorbance at $260 \mathrm{~nm}$ and $150 \mathrm{ng}$ of DNA was used for the PCR. Two PCR reactions per sample were performed: one to detect Cre gene product and another one to detect Flox gene product. For Cre PCR, the primers used were as: oIMR 3621 (F: 5' to 3' CGT GAT CTG CAA CTC CAG TC) and oIMR 9074 (R: 5' to 3' AGG CAA ATT TTG GTG TAC GG). For Flox PCR, the primers used were as: oIMR 5116 (F: 5' to $3^{\prime}$ GCT TTG TTA TCC CAG CTG GCG C) and oIMR 5115 (R: 5' to 3' TTC TCA CCA GTG CAT AGG GCT CTG). PCR was performed in $12 \mu \mathrm{l}$ of reaction volume using the kit (KAPA2G Robust Hotstart Readymix PCR kit) using an applied biosystems thermal cycler (GeneAmp PCR system 2700). The thermal cycler conditions were set according to the genotyping protocol for mice stock \# 005740 by the Jackson Lab and the PCR products separated using $0.7 \%$ agarose gel electrophoresis.

\section{Tamoxifen Injection}

Tamoxifen base (T5648, Sigma) was dissolved in peanut oil $(10 \mathrm{mg} / \mathrm{ml})$ and was given as $40 \mathrm{mg} / \mathrm{kg}$ as described by Andersson et al. (2010). Eight-week-old Ppif ${ }^{\mathrm{fl} / \mathrm{Fl}}-\mathrm{Cre}^{/+}$mice (Flox/flox/cre, Cyp-D CKO) and Ppif ${ }^{\mathrm{fl} / \mathrm{Fl}}-\mathrm{Cre}^{--}$mice (Flox/flox, control mice) received intraperitoneally $1 \mathrm{mg}$ of tamoxifen per day for five consecutive days. Administration of tamoxifen induces activation of the ubiquitously expressed $\mathrm{Cre}^{\mathrm{ERT2}}$ recombinase, which stimulates recombination and excision of floxed Cyp-D in all tissues. Since Ppif ${ }^{\mathrm{F} / \mathrm{Fl}}$ mice do not contain Cre gene, they will not undergo Cyp-D recombination and serve as control.

\section{Treadmill Running}

After 2 weeks of completion of tamoxifen injections (i.e., on day 19 as shown in Figure 1), treadmill running was started to assess effects on oxygen metabolism (i.e., $\mathrm{VO}_{2}, \mathrm{VCO}_{2}$, and
RER) in CKO and control mice at rest and during treadmill exercise using a calorimeter (Oxymax System, Columbus Instruments).

Treadmill running had two components: (1) Training protocol and (2) Treadmill exercise protocol.

\section{Treadmill Training Protocol}

It was performed as previously described (Radhakrishnan et al., 2019). Each mouse was first subjected to a 3-day habituation period. On day 1 , the mouse ran $10 \mathrm{~min}$ at $10 \mathrm{~m} / \mathrm{min}$ without inclination; on day 2 , the mouse ran $10 \mathrm{~min}$ at $10 \mathrm{~m} / \mathrm{min}$ with 20 degree inclination; and on day 3, the mouse ran $20 \mathrm{~min}$ at the same speed and inclination. An electrical grid placed at the start of the treadmill ramp delivered an aversive shock when the mouse stopped running and slid into the grid to elicit avoidance of subsequent shocks by running (i.e., avoidance conditioning). The mouse was discarded from the study if more than three shocks in one session were required to promote running.

\section{Treadmill Exercise Protocol}

It was performed as previously described (Pederson et al., 2005; Radhakrishnan et al., 2019). Each mouse ran for a total of five sessions with the treadmill at a constant $20^{\circ}$ inclination. At the start of each session, the mouse was allowed to acclimate in the chamber for $30 \mathrm{~min}$ before running. Baseline measurements were obtained between min 25 and 30 . The treadmill was then started at $10 \mathrm{~m} / \mathrm{min}$ and its speed increased by $2 \mathrm{~m} / \mathrm{min}$ every $4 \mathrm{~min}$ to a maximum of $26 \mathrm{~m} / \mathrm{min}$ for session one and to $30 \mathrm{~m} / \mathrm{min}$ in sessions two through five. The mouse was allowed to run at the maximum speed for up to $90 \mathrm{~min}$ or until exhaustion. The strength of the shock was kept at $2 \mathrm{~mA}$ for the first $30 \mathrm{~min}$ running at maximum speed, after which the current was reduced to $0.5 \mathrm{~mA}$. Exhaustion was defined as sliding into the shock grid and sustaining a shock (0.5 or $2 \mathrm{~mA}$ ) for a minimum of $2 \mathrm{~s}$ for the third time or sustaining a single shock of $\geq 5 \mathrm{~s}$ (instead of running). The investigators were blind to the mice genotype. The treadmill was cleaned between animals with $70 \%$ ethanol, wiped with napkins, and air dried. Each running session was completed by the mice cohorts (20 mice total) in 4 days. There was a 3 -day resting period afterward resulting in a week for completion of one running session by the mice cohorts. It took a total of 32 days to complete the five sessions.

\section{$\mathrm{VO}_{2}, \mathrm{VCO}_{2}$, and Derived Measurements}

The measurements were performed as previously described (Radhakrishnan et al., 2019). Power (Joules/min) was calculated by dividing work performed (Joules) by time spent on treadmill (min). Fuel utilization (carbohydrate, $\mathrm{g} / \mathrm{min}$ and fat, $\mathrm{g} / \mathrm{min}$ ) was calculated using $\mathrm{VO}_{2}$ and $\mathrm{VCO}_{2}$ data as previously described (Radhakrishnan et al., 2019) according to Carbohydrate (mg/ $\min )=\left[-3.226 \mathrm{~g} \quad\right.$ carbohydrate $/ \mathrm{LO}_{2} \times \mathrm{VO}_{2} \quad(\mathrm{~L} / \mathrm{min})+4.585 \mathrm{~g}$ carbohydrate $\left./ \mathrm{LCO}_{2} \times \mathrm{VCO}_{2} \quad(\mathrm{~L} / \mathrm{min})\right] \times 1,000 ;$ Fat $\quad(\mathrm{mg} /$ $\mathrm{min})=\left[1.695 \mathrm{~g} \mathrm{fat} / \mathrm{LO}_{2} \times \mathrm{VO}_{2}(\mathrm{~L} / \mathrm{min})-1.701 \mathrm{~g} \mathrm{fat} / \mathrm{LCO}_{2} \times \mathrm{VCO}_{2}\right.$ $(\mathrm{L} / \mathrm{min})] \times 1,000$ (Petrosino et al., 2016) 


\section{A}

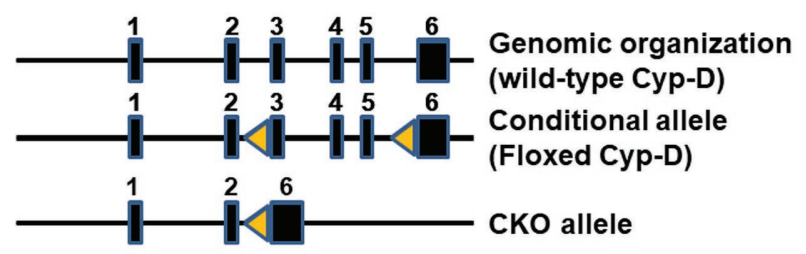

B

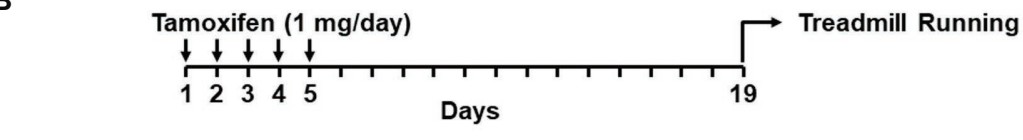

C

D

E
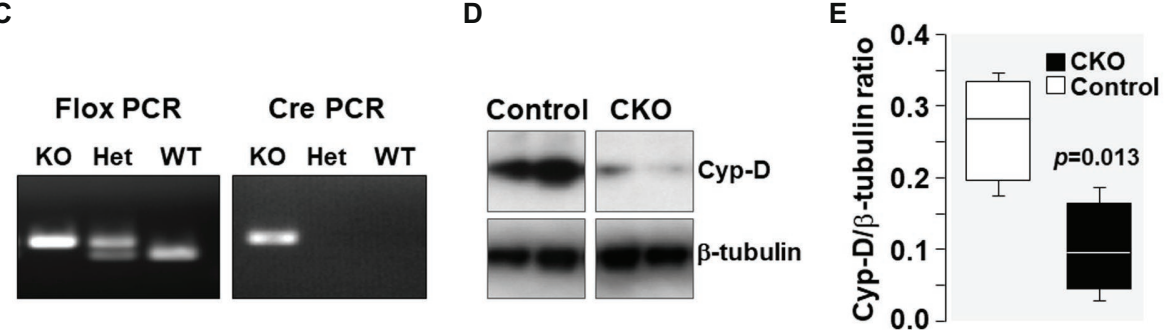

FIGURE 1 | (A) Genomic organization of Cyp-D encoding ppif gene in mouse chromosome 14 is shown. The ppif gene has six exons which are represented by closed boxes. Genomic organization of floxed Cyp-D in which exons 3-5 has flanking lox-P sites (yellow triangles) is shown as conditional allele. The floxed Cyp-D mouse was generated by Stanley J. Korsmeyer's lab. Genomic organization of conditional knockout (CKO) allele showing deletion of exons $3-5$. (B) Schematic demonstrating time of tamoxifen treatment. (C) Ppif ${ }^{\mathrm{F} /+}-\mathrm{Cre} /+$ mice pups were genotyped to confirm the presence of Ppif ${ }^{\mathrm{F} /+}$ and $\mathrm{Cre}$ genes. (D) Western blot showing Cyp-D protein in skeletal muscle tissue homogenates of control and cyclophilin-D conditional knockout (CKO) mice. (E) Box plots denote densitometry of Cyp-D normalized to $\beta$-tubulin.

\section{Western Blotting}

The Western blotting was performed as previously described (Radhakrishnan et al., 2019).

\section{Statistical Analysis}

Linear mixed effect model was used to assess the effect of genotype and running sessions and their interaction on metabolic variables treating running sessions as discrete variable (SPSS 24.0, IBM Corp.; Armonk, NY, United States). Student's $t$-test was used to assess (i) the effect of genotype on metabolic variables collected at baseline and at exercise and (ii) the effect of genotype on molecular signaling implementing t-test (when normality test passed) and Mann-Whitney Rank Sum test (when normality test failed; SigmaPlot v.12.5). Strength of relationships between variables was assessed by linear regression and Pearson's product moment correlation analysis (SigmaPlot v.12.5). Data are presented as means \pm SD in the text and means \pm SEM in the figures. A two-tail value of $p \leq 0.05$ was considered significant.

\section{RESULTS}

\section{Confirmation of Cyp-D Knockout}

Genomic organization of the mouse genome at chromosome 14 and the strategy for conditional $\mathrm{KO}$ generation is shown in Figure 1. As mentioned, $\mathrm{Ppif}{ }^{\mathrm{F} /++}-\mathrm{Cre}^{/+}$mice have Ppif allele flanked between two lox-P sites which upon tamoxifen injection will undergo Cre-mediated recombination resulting in deletion of the Ppif allele. Ppif ${ }^{\mathrm{Fl} /+}-\mathrm{Cre}^{/+}$mice pups were genotyped to confirm the presence of $\mathrm{Ppif}^{\mathrm{Fl} /+}$ and Cre genes. Results showed that the products of expected size were generated by flox PCR ( $400 \mathrm{bp}$ ) and by Cre PCR (200bp). Tamoxifen injection resulted in Cre-mediated deletion of lox-P flanked Ppif allele and was confirmed by Western blotting detection of Cyp-D levels in mice skeletal muscle tissue homogenates (Figure 1).

\section{Effect of Conditional Cyp-D Knockout on $\mathrm{VO}_{2}, \mathrm{VCO}_{2}$, and RER}

At their first treadmill running session, as shown in Figure 2A, CKO mice and controls had comparable $\mathrm{VO}_{2}, \mathrm{VCO}_{2}$, and RER. However, with subsequent sessions, the $\mathrm{VO}_{2}$ decreased in $\mathrm{CKO}$ mice without a significant change in $\mathrm{VCO}_{2}$. The lower $\mathrm{VO}_{2}$ for a given $\mathrm{VCO}_{2}$ manifested in a significantly higher RER that remained statistically significant for most of the sessions (Figure 2A). This behavior is consistent with a metabolic adaptation induced by exercise training in $\mathrm{CKO}$ mice.

All individual $\mathrm{VO}_{2}, \mathrm{VCO}_{2}$, and RER measurements obtained at baseline - before starting treadmill exercise - combining the five sessions are shown in Figure 2B. CKO mice had a lower $\mathrm{VO}_{2}(p=0.003)$ and a lower $\mathrm{VCO}_{2}(p=0.014)$ but of lesser magnitude yielding a slightly higher RER which did not attain statistical significance $(p=0.201)$.

All individual $\mathrm{VO}_{2}, \mathrm{VCO}_{2}$, and RER measurements obtained during treadmill running combining the five sessions are shown 
A

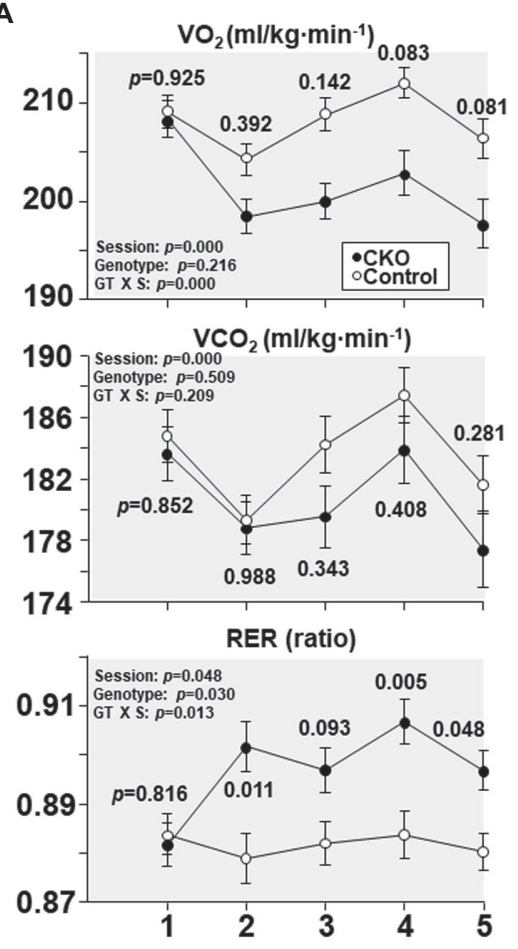

\section{B}

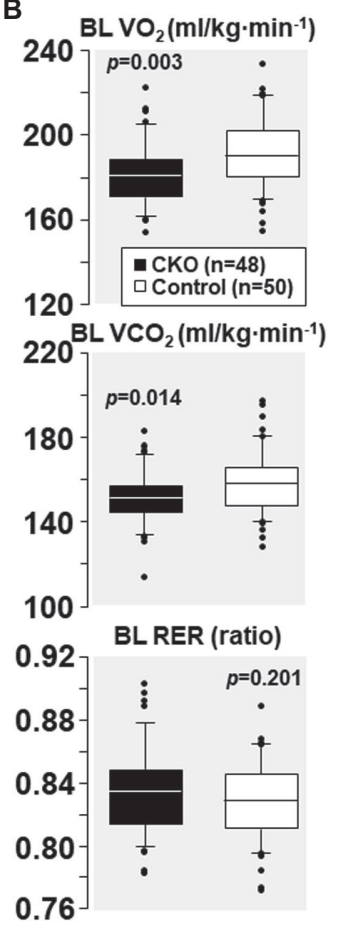

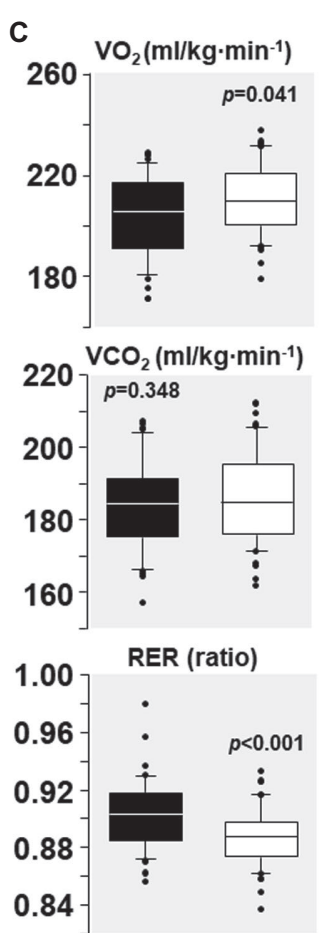

FIGURE 2 | (A) Oxygen consumption $\left(\mathrm{VO}_{2}\right)$, carbon dioxide production $\left(\mathrm{VCO}_{2}\right)$, and respiratory exchange ratio (RER) of cyclophilin-D conditional knockout (CKO; $n=10)$ and control mice $(n=10)$ at five running sessions. Values are shown as mean \pm SEM. Differences were analyzed by a linear mixed-effects model. Data from one $\mathrm{CKO}$ mouse were lost at running sessions four and five due to refusal of running. (B) All individual oxygen consumption $\left(\mathrm{VO}_{2}\right)$, carbon dioxide production $\left(\mathrm{VCO}_{2}\right)$, and respiratory exchange ratio (RER) measurements obtained under baseline (BL) conditions in cyclophilin-D conditional knockout (CKO) and control mice are shown as box plots, comparing the groups using t-test. (C) Box plots depicting all individual data points of oxygen consumption $\left(\mathrm{VO}_{2}\right)$, carbon dioxide production $\left(\mathrm{VCO}_{2}\right.$ ), and respiratory exchange ratio (RER) during exercise at five running sessions by conditional cyclophilin-D knockout (CKO) and control mice. Differences were analyzed by t-test.

in Figure 2C. CKO mice also exhibited a lower $\mathrm{VO}_{2}(p=0.04)$ but without a significant difference in $\mathrm{VCO}_{2}(p=0.348)$, yet with a highly significant increase in RER $(p<0.001)$.

\section{Effect of Conditional Cyp-D Knockout on Exercise Capacity}

From the first and the subsequent sessions, CKO mice had a higher power and power $/ \mathrm{VO}_{2}$ but did not attain statistical significance (Figure 3). Yet, when all individual power and power $/ \mathrm{VO}_{2}$ ratio throughout the five running sessions were combined, $\mathrm{CKO}$ mice had a statistically significant higher power and power $/ \mathrm{VO}_{2}$ (Figure 3), consistent with increased oxygen utilization efficiency.

\section{Effect of Conditional Cyp-D Knockout on Fuel Utilization}

As shown in Figure $\mathbf{4 A}$, at their first running session, the fuel utilization was comparable between CKO mice and controls. Yet, with subsequent running sessions, carbohydrate utilization was higher in CKO mice but the difference was not statistically significant. Likewise, fat utilization was also comparable between the genotypes at the first running session. However, with subsequent running sessions, fat utilization was lower in $\mathrm{CKO}$ mice attaining statistical significance in most of the running sessions (Figure 4A).

All individual carbohydrate and fat utilization at baseline before starting treadmill exercise - combining the five sessions demonstrated comparable carbohydrate and fat utilization in $\mathrm{CKO}$ mice and controls (Figure 4B). All individual carbohydrate and fat utilization during exercise throughout the five running sessions showed increased carbohydrate and decreased fat utilization in $\mathrm{CKO}$ mice (Figure 4C).

\section{Effect of Conditional Cyp-D Knockout on AMPK-TBC1D1 Nexus and Its Downstream Signaling}

AMPK phosphorylation at Thr 172 - indicative of its activation was increased by $64 \%$ and TBC1D1 phosphorylation Ser $660-$ indicative of its inactivation - was increased by $55 \%$ in $\mathrm{CKO}$ mice skeletal muscles compared to control (Figure 5). Analysis of relationship between pAMPK and pTBC1D1 (Figure 5) showed a positive correlation which did not attain statistical significance $(\mathrm{R}=0.639 ; p=0.08)$.

PGC- $1 \alpha$ levels increased by $162 \%$ and GLUT4 levels increased by $103 \%$ in $\mathrm{CKO}$ mice skeletal muscles compared to control (Figure 6). Analysis of relationship between GLUT4 and AMPK 


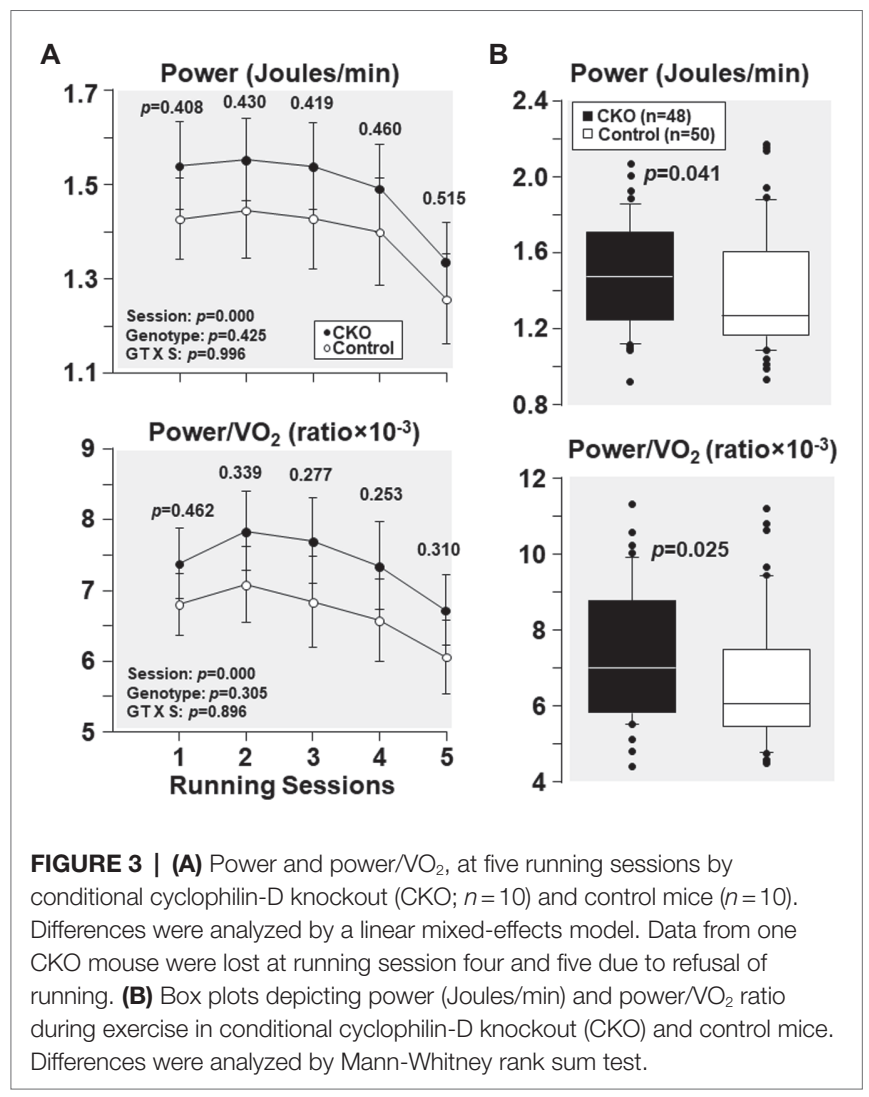

demonstrated a positive correlation without statistical significance $(\mathrm{R}=0.686 ; p=0.061)$.

The power $/ \mathrm{VO}_{2}$ exhibited a positive correlation with the $\mathrm{pAMPK} / \beta$-tubulin ratio $(\mathrm{R}=0.753 ; p=0.03)$ and the PGC- $1 \alpha / \beta$ tubulin ratio $(\mathrm{R}=0.708 ; p=0.04$; Figure 7$)$.

\section{DISCUSSION}

Consistent with our previous studies showing that Cyp-D ablation resulted in downregulation of $\mathrm{VO}_{2}$ in HEK $293 \mathrm{~T}$ cells and in constitutive $\mathrm{KO}$ mice, with increased oxygen utilization efficiency in the latter, the present studies demonstrate for the first time that conditional Cyp-D ablation in adult mice also reduces systemic $\mathrm{VO}_{2}$ and increases oxygen utilization efficiency. Reduction in $\mathrm{VO}_{2}$ was accompanied by higher power during treadmill exercise for a given $\mathrm{VO}_{2}$ and was accompanied by increased RER consistent with preferential utilization of glucose over fat. We also found direct evidence of AMPK activation with concomitant TBC1D1 inactivation and increased GLUT4 expression in skeletal muscle tissues of Cyp-D CKO mice after exercise. Taken together, these studies demonstrate that conditional Cyp-D ablation in adults leads to an adaptive response that improves oxygen utilization efficiency and exercise capacity associated with activation of AMPK signaling, an effect that invites work on options for pharmacologically recapitulating the effect, which would be beneficial under oxygen limiting conditions.

\section{Conditional Cyp-D Ablation Reduces $\mathbf{V O}_{2}$}

We have previously reported in HEK $293 \mathrm{~T}$ cells that Cyp-D silencing results in downregulation of mitochondrial transcripts and concomitant reduction in cellular oxygen consumption (Radhakrishnan et al., 2015). Further studies in constitutive Cyp-D KO mice demonstrated downregulation of $\mathrm{VO}_{2}$ at rest and during endurance exercise (Radhakrishnan et al., 2019). In the present studies, we demonstrated that $\mathrm{VO}_{2}$ in conditional Cyp-D KO adult mice at rest and during exercise was comparable to control mice in the first running session and decreased only with subsequent sessions. Decrease in $\mathrm{VO}_{2}$ was accompanied by increased RER indicative of an adaptational response and training effect in $\mathrm{CKO}$ mice.

\section{Conditional Cyp-D Ablation Improves Exercise Capacity and Induces Metabolic Switch With Concomitant Activation of AMPK-TBC1D1 Signaling Nexus}

During exercise, in contracting muscles, activation of AMPK occurs consequent to AMP accumulation as ATP consumption increases (Winder and Hardie, 1996). AMPK activation occurs mainly through activation of the upstream liver kinase B1(kinase LKB1) through Thr172 phosphorylation of its catalytic domain in response to increased cellular AMP:ATP ratio (Hawley et al., 2003; Woods et al., 2003). Activated AMPK inactivates its downstream target TBC1D1 (Kramer et al., 2006; Treebak et al., 2006; Taylor et al., 2008; Ferrari et al., 2019; de Wendt et al., 2021) by phosphorylation (an interaction also known as "AMPKTBC1D1 signaling nexus"; Chavez et al., 2008; Taylor et al., 2008; Vichaiwong et al., 2010; Treebak et al., 2014; Kjøbsted et al., 2016; Ferrari et al., 2019). TBC1D1 inactivation during exercise has been reported in mouse skeletal muscles (Taylor et al., 2008; Vichaiwong et al., 2010; de Wendt et al., 2021) and in human skeletal muscles (Jessen et al., 2011; Tobias et al., 2020). Activation of AMPK-TBC1D1 signaling nexus results in increased GLUT4 expression and translocation from the cytosol to the plasma membrane enabling increased glucose uptake. Glucose enters the glycolytic pathway and is metabolized in the cytosol to pyruvate and then to lactate by lactate dehydrogenase $(\mathrm{LDH})$. The conversion of pyruvate to lactate is favored over alternative pyruvate fates given the nearequilibrium nature of the $\mathrm{LDH}$ reaction (Lambeth and Kushmerick, 2002) and a much higher activity than regulatory enzymes of the glycolytic and oxidative pathways (Rogatzki et al., 2015). Lactate then enters the mitochondria where it is converted to pyruvate by the $\mathrm{LDH}$ present on the mitochondrial inner membrane (Hashimoto et al., 2006; Gladden, 2008). Pyruvate is then converted to acetyl coA and metabolized in the Krebs cycle. Thus, the AMPK-TBC1D1 signaling nexus increases glucose uptake and enhances glucose utilization through glycolysis and the Krebs cycle. Consistent with this effect, we observed in the present study activation of AMPK-TBC1D1 signaling nexus (Figure 5) and increased GLUT4 expression (Figure 6) in $\mathrm{CKO}$ mice after exercise. Consistently, several other studies have also showed increased expression of skeletal muscle GLUT4 in rats and human after exercise (Ivy et al., 1999; 


\section{A}

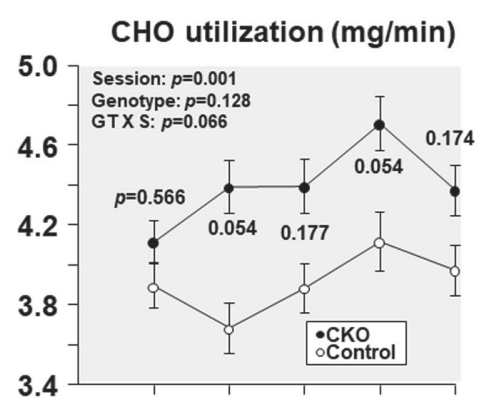

3.4 Fat utilization $(\mathrm{mg} / \mathrm{min})$

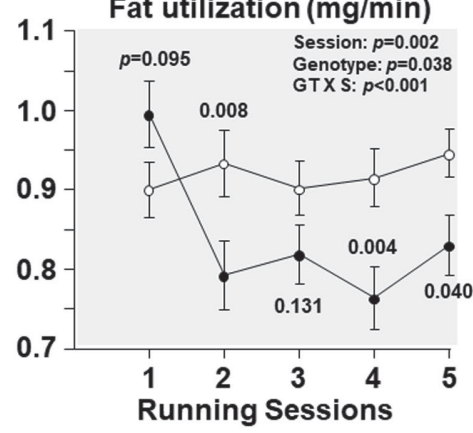

B

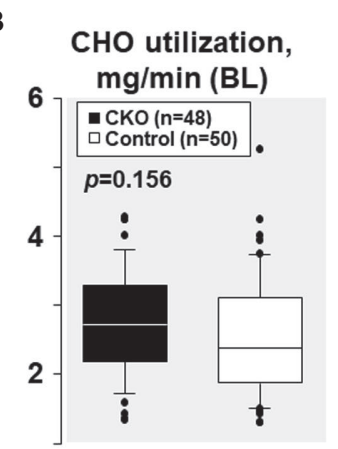

Fat utilization, $\mathrm{mg} / \mathrm{min}$ (BL)

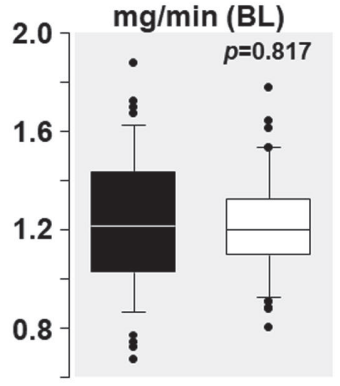

C

CHO utilization,

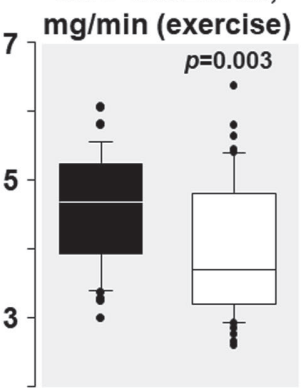

Fat utilization,

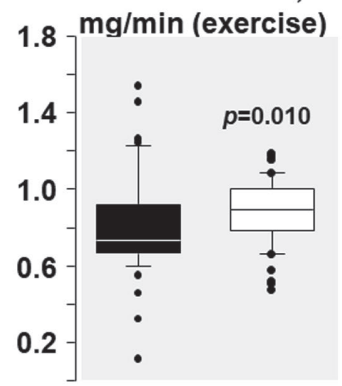

FIGURE 4 | (A) Carbohydrate (CHO) and fat utilization at five running sessions by conditional cyclophilin-D knockout (CKO; $n=10)$ and control mice ( $n=10)$. Differences were analyzed by a linear mixed-effects model. Data from one CKO mouse were lost at running session four and five due to refusal of running. (B) Box plots depicting estimation of carbohydrate and fat utilization by conditional cyclophilin-D knockout (CKO) and control mice at baseline (BL) and (C) during exercise (all individual data points of all five running sessions). Differences were analyzed by t-test when normality test passed (CHO utilization) and by Mann-Whitney Rank Sum test when normality test failed (Fat utilization).

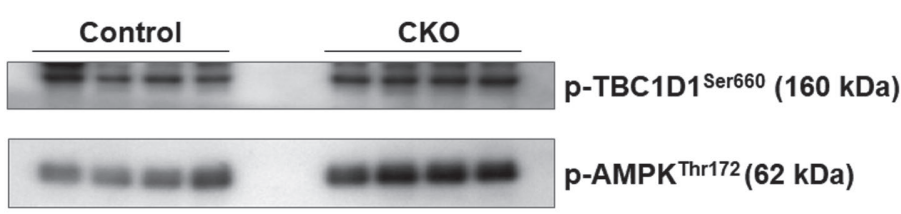

$\beta$ - $\beta$-tubulin (55 kDa)

PTBC1D1/ $\beta$-tubulin (ratio)

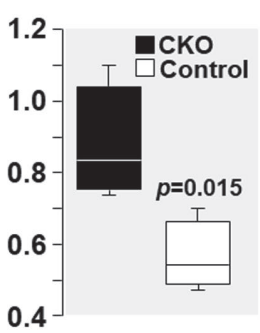

PAMPK/Btubulin (ratio)

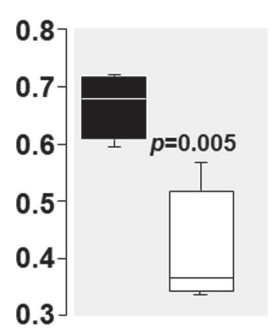

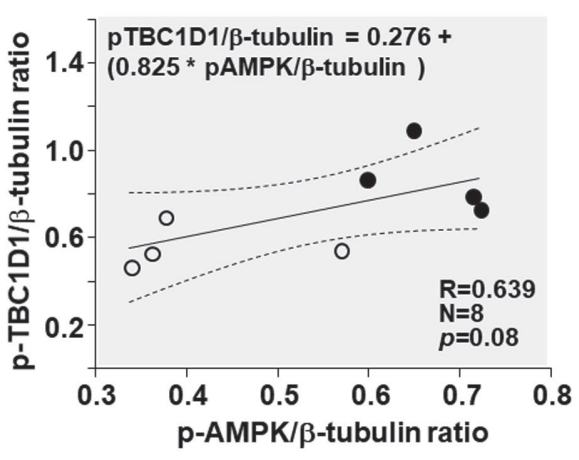

FIGURE 5 | Western blots demonstrating levels of AMPK phosphorylation at threonine 172 (Thr172), TBC1D1 phosphorylation at serine 660 (Ser 660), and $\beta$-tubulin (loading control) in mice skeletal muscles after exercise. Box plots denote densitometry of PAMPK and pTBC1D1 normalized to $\beta$-tubulin. $N=4$ from each group. Relationship between pAMPK/ $\beta$-tubulin ratio and pTBC1D1/ $\beta$-tubulin ratio is shown. The regression line is shown with the corresponding $95 \%$ confidence interval. 

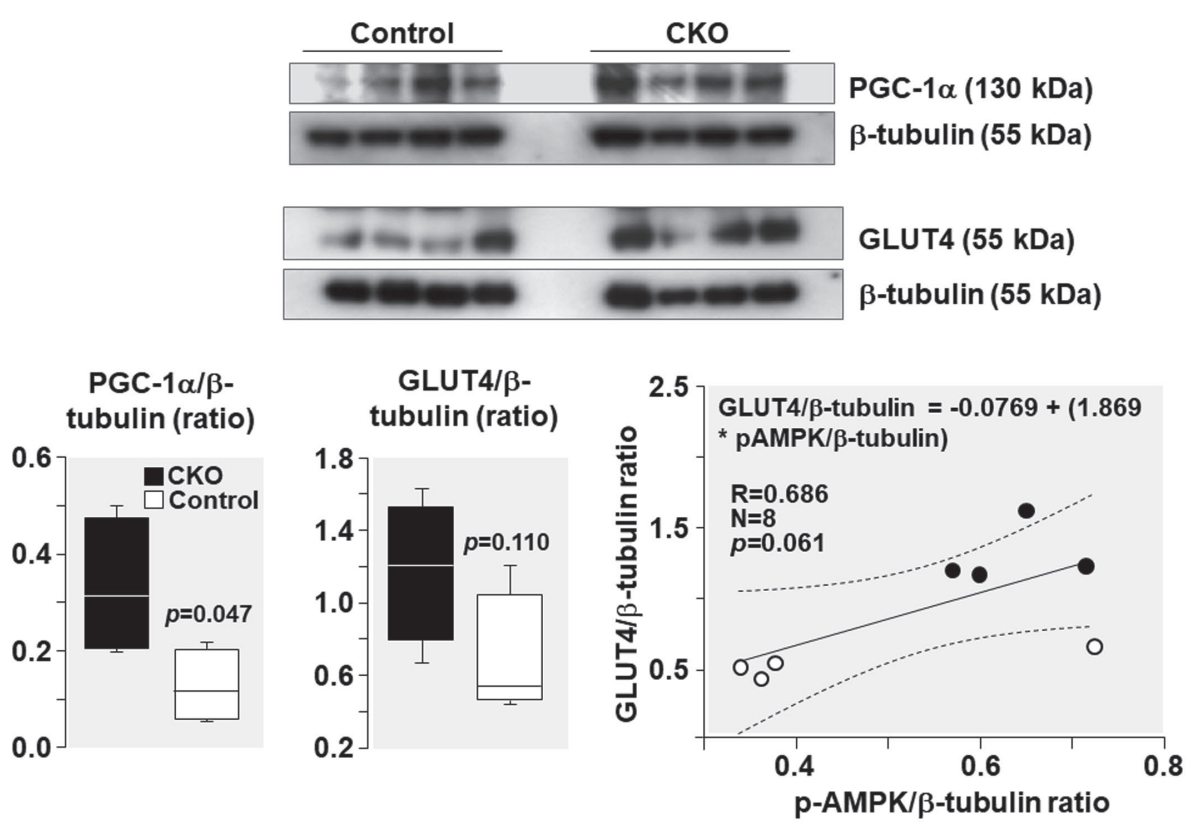

FIGURE 6 | Western blots demonstrating levels of PGC-1 $\alpha$, GLUT4, and $\beta$-tubulin (loading control) in mice skeletal muscle after exercise. Box plots denote densitometry of PGC- $1 \alpha$ and GLUT4 normalized to $\beta$-tubulin. $N=4$ from each group. Relationship between pAMPK/ $\beta$-tubulin ratio and GLUT4/ $\beta$-tubulin ratio is shown. The regression line is shown with the corresponding $95 \%$ confidence interval.
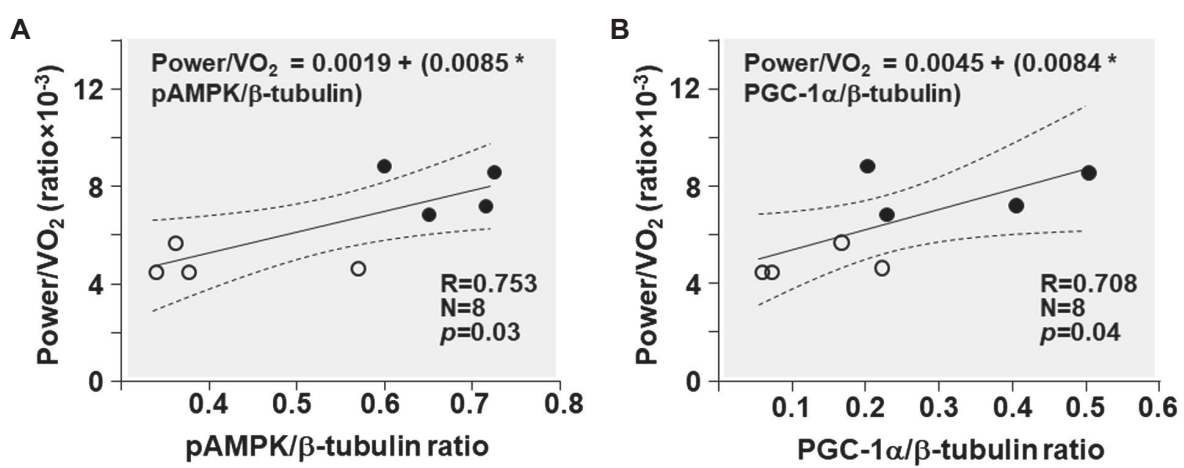

FIGURE 7 | Shown are the relationships between (A) pAMPK/ $\beta$-tubulin ratio and power $\mathrm{NO}_{2}$ ratio and (B) PGC-1 $\alpha / \beta$-tubulin ratio and power $/ \mathrm{NO}{ }_{2}$ ratio in $\mathrm{CKO}$ mice (black circles) and controls (white circles). The regression line is shown with the corresponding 95\% confidence interval.

Kuo et al., 1999; MacLean et al., 2000; Flores-Opazo et al., 2020). Increased GLUT4 expression has been correlated with increased glucose transport and utilization (Kern et al., 1990; Ivy et al., 1999; MacLean et al., 2000).

In addition, AMPK activation also increases the expression of PGC-1 $\alpha$ (as also shown in Figure 6). The effect occurs by phosphorylation at its threonine-177 and serine-538 residues prompting self-dependent activation of its promoter and gene expression (Jäger et al., 2007). PGC-1 $\alpha$ further increases GLUT4 expression and thereby glucose uptake (Jäger et al., 2007). In addition, PGC- $1 \alpha$ activates mitochondrial biogenesis and other pathways, which could improve the exercise capacity after training. Several lines of evidence suggest that PGC- $1 \alpha$ is a key factor in mediating exercise training induced adaptations in mitochondria
(Olesen et al., 2010) in response to AMPK activation. For example, AICAR - the AMPK activator - improves exercise capacity in mice with concomitant increase in PGC-1 $\alpha$ gene expression (Narkar et al., 2008). In addition, AICAR injections fail to increase levels of mitochondrial and metabolic proteins in skeletal muscles of PGC-1 $\alpha$ KO mice (Leick et al., 2010). Thus, AMPK in CKO mice could induce additional adaptive responses, including mitochondrial biogenesis via PGC-1 $\alpha$, leading to enhanced exercise capacity.

In our previous studies in constitutive Cyp-D KO mice, the aforementioned metabolic effects were associated with downregulation of electron transport complexes I and IV activities consequent to reduced expression of subunits encoded by the mitochondrial heavy strand promoter 2 (Radhakrishnan et al., 2015, 2019). The expression of these subunits 
requires the interaction of Cyp-D with the mitochondrial transcription factors B1 and B2 (Radhakrishnan et al., 2015). The reduced activity of electron transport complexes I and IV would create an "energy-stress" (Gowans et al., 2013) during exercise prompting an increase in AMP:ATP ratio and the consequent activation of the AMPK-TBC1D1 signaling nexus. Other studies have also reported complex I inhibition as the mechanism of AMPK activation. In fact, the mechanism of pharmacological activation of AMPK by metformin involves electron complex I inhibition (Owen et al., 2000; Zhou et al., 2001; Mohsin et al., 2019). However, metformin abrogates exercise-mediated increase in skeletal muscle mitochondrial respiration (Konopka et al., 2019).

In our previous study in constitutive Cyp-D KO mice, we have documented a metabolic shift - i.e., increased glucose utilization over fat. Tavecchio et al. (2015) have documented in their study that Cyp-D constitutive $\mathrm{KO}$ mice had increased transcription of genes involved in glucose metabolism resulting in increased glucose utilization and impaired fatty acid utilization. In the present study in conditional Cyp-D KO mice, we have documented a similar adaptive response. In the present study, AMPK activation was accompanied by increased glucose utilization over fatty acids (as shown in Figure 4) that is energetically advantageous. Glucose oxidation per mole yields 6.3 moles of ATP, whereas fatty acid yields only 5.6 moles of ATP (Opie, 1991; Stanley and Chandler, 2002) resulting in a $12.5 \%$ increase in energy production with glucose oxidation. Thus, this metabolic switch enhances oxygen utilization efficiency during exercise and improves exercise capacity as evidenced by the significant increase in power during treadmill exercise for given oxygen consumed (power/ $/ \mathrm{VO}_{2}$ ratio; as shown in Figure 3).

\section{Comparison Between Constitutive and Conditional Cyp-D KO}

Both constitutive and conditional Cyp-D KO had their Cyp-D knocked-out in all tissues. Cyp-D ablation was carried out at an embryonic stage in constitutive $\mathrm{KO}$, whereas Cyp-D ablation was carried out at the adult stage in $\mathrm{CKO}$ mice. Yet, the $\mathrm{CKO}$ mice displayed similar effects $-\mathrm{VO}_{2}$ downregulation and metabolic switch - as that of constitutive KO but of lesser magnitude. The metabolic switch in $\mathrm{CKO}$ mice was prominent at the second running session and thereafter, as documented by increased glucose utilization over fatty acids (Figure 4). The first and second running sessions were performed at the third and the fourth week, respectively, after tamoxifen treatment for 5 days. This hints to the possibility that the metabolic switch can be triggered probably only 4 weeks after completion of pharmacological interventions. The time delay for this metabolic switch - we rationalize - includes the time required for cre/lox-P recombination-dependent Cyp-D ablation and subsequent respiratory chain downregulation. In addition, exercise training induced mitochondrial adaptations involving

\section{REFERENCES}

Andersson, K. B., Winer, L. H., Mørk, H. K., Molkentin, J. D., and Jaisser, F. (2010). Tamoxifen administration routes and dosage for inducible Cre-
PGC- $1 \alpha$ could also have played role in improving the exercise capacity. These results point to the fact that selectively destabilizing interaction between Cyp-D and mitochondrial transcription factors to reduce oxygen consumption without affecting other functions of Cyp-D in adults could have potential positive impact on oxygen utilization efficiency and it could be highly beneficial under oxygen limiting conditions.

\section{Conclusion}

Conditional Cyp-D ablation reduced $\mathrm{VO}_{2}$ and increased power/ $\mathrm{VO}_{2}$ ratio, demonstrating increased oxygen utilization efficiency consistent with our previous work in constitutive $\mathrm{KO}$ mice. Conditional Cyp-D ablation also triggered the adaptive response which involved a metabolic switch toward increased glucose utilization with concomitant activation of AMPK-TBC1D1 signaling nexus and increased GLUT4 expression in skeletal muscles after exercise. Our study raises the possibility that the metabolic switch can probably be triggered in 4 weeks after completion of pharmacological intervention. Developing tools for modulation of this adaptive response could be beneficial in a myriad of physiological and clinical conditions where oxygen availability is limited.

\section{DATA AVAILABILITY STATEMENT}

The raw data supporting the conclusions of this article will be made available by the authors, without undue reservation.

\section{ETHICS STATEMENT}

The animal study was reviewed and approved by the Institutional Animal Care and Use Committee at Rosalind Franklin University of Medicine and Science (Protocol B17-20) and by the Edward Hines VA Hospital Institutional Animal Care and Use Committee (Protocol H17-014).

\section{AUTHOR CONTRIBUTIONS}

JR and RJG conceived and designed the research and wrote the manuscript and agreed on the final version. JR performed the experiments with the assistance of $\mathrm{AB}$. $\mathrm{AB}$ developed the software necessary for the experiments and data analysis. JR and $\mathrm{AB}$ analyzed the data. All authors contributed to the article and approved the submitted version.

\section{FUNDING}

We are thankful for the funding support by the VA Merit Review Award (1 I01 BX002771-01) to RJG.

mediated gene disruption in mouse hearts. Transgenic Res. 19, 715-725. doi: $10.1007 /$ s11248-009-9342-4

Chavez, J. A., Roach, W. G., Keller, S. R., Lane, W. S., and Lienhard, G. E. (2008). Inhibition of GLUT4 translocation by Tbc1d1, a Rab GTPase-activating 
protein abundant in skeletal muscle, is partially relieved by AMP-activated protein kinase activation. J. Biol. Chem. 283, 9187-9195. doi: 10.1074/jbc. M708934200

de Wendt, C., Espelage, L., Eickelschulte, S., Springer, C., Toska, L., Scheel, A., et al. (2021). Contraction-mediated glucose transport in skeletal muscle is regulated by a framework of AMPK, TBC1D1/4 and Rac1. Diabetes doi: 10.2337/db21-0587 [Epub ahead of print]

Elrod, J. W., Wong, R., Mishra, S., Vagnozzi, R. J., Sakthievel, B., Goonasekera, S. A., et al. (2010). Cyclophilin D controls mitochondrial pore-dependent $\mathrm{Ca}(2+)$ exchange, metabolic flexibility, and propensity for heart failure in mice. $J$. Clin. Invest. 120, 3680-3687. doi: 10.1172/JCI43171

Ferrari, F., Bock, P. M., Motta, M. T., and Helal, L. (2019). Biochemical and molecular mechanisms of glucose uptake stimulated by physical exercise in insulin resistance state: role of inflammation. Arq. Bras. Cardiol. 113, 1139-1148. doi: $10.5935 / a b c .20190224$

Flores-Opazo, M., McGee, S. L., and Hargreaves, M. (2020). Exercise and GLUT4. Exerc. Sport Sci. Rev. 48, 110-118. doi: 10.1249/JES.0000000000000224

Gladden, L. B. (2008). 200th anniversary of lactate research in muscle. Exerc. Sport Sci. Rev. 36, 109-115. doi: 10.1097/JES.0b013e31817c0038

Gowans, G. J., Hawley, S. A., Ross, F. A., and Hardie, D. G. (2013). AMP is a true physiological regulator of AMP-activated protein kinase by both allosteric activation and enhancing net phosphorylation. Cell Metab. 18, 556-566. doi: 10.1016/j.cmet.2013.08.019

Hashimoto, T., Hussien, R., and Brooks, G. A. (2006). Colocalization of MCT1, CD147, and LDH in mitochondrial inner membrane of L6 muscle cells: evidence of a mitochondrial lactate oxidation complex. Am. J. Physiol. Endocrinol. Metab. 290, E1237-E1244. doi: 10.1152/ajpendo.00594. 2005

Hawley, S. A., Boudeau, J., Reid, J. L., Mustard, K. J., Udd, L., Mäkelä, T. P., et al. (2003). Complexes between the LKB1 tumor suppressor, STRAD alpha/ beta and MO25 alpha/beta are upstream kinases in the AMP-activated protein kinase cascade. J. Biol. 2:28. doi: 10.1186/1475-4924-2-28

Ivy, J. L., Zderic, T. W., and Fogt, D. L. (1999). Prevention and treatment of non-insulin-dependent diabetes mellitus. Exerc. Sport Sci. Rev. 27, 1-35.

Jäger, S., Handschin, C., St-Pierre, J., and Spiegelman, B. M. (2007). AMPactivated protein kinase (AMPK) action in skeletal muscle via direct phosphorylation of PGC-1alpha. Proc. Natl. Acad. Sci. U. S. A. 104, 12017-12022. doi: 10.1073/pnas.0705070104

Jessen, N., An, D., Lihn, A. S., Nygren, J., Hirshman, M. F., Thorell, A., et al. (2011). Exercise increases TBC1D1 phosphorylation in human skeletal muscle. Am. J. Physiol. Endocrinol. Metab. 301, E164-E171. doi: 10.1152/ ajpendo.00042.2011

Kern, M., Wells, J. A., Stephens, J. M., Elton, C. W., Friedman, J. E., Tapscott, E. B., et al. (1990). Insulin responsiveness in skeletal muscle is determined by glucose transporter (Glut4) protein level. Biochem. J. 270, 397-400. doi: 10.1042/bj2700397

Kjøbsted, R., Pedersen, A. J. T., Hingst, J. R., Sabaratnam, R., Birk, J. B., Kristensen, J. M., et al. (2016). Intact regulation of the AMPK signaling network in response to exercise and insulin in skeletal muscle of male patients with type 2 diabetes: illumination of AMPK activation in recovery from exercise. Diabetes 65, 1219-1230. doi: 10.2337/db15-1034

Konopka, A. R., Laurin, J. L., Schoenberg, H. M., Reid, J. J., Castor, W. M., Wolff, C. A., et al. (2019). Metformin inhibits mitochondrial adaptations to aerobic exercise training in older adults. Aging Cell 18:e12880. doi: 10.1111/ acel. 12880

Kramer, H. F., Witczak, C. A., Fujii, N., Jessen, N., Taylor, E. B., Arnolds, D. E., et al. (2006). Distinct signals regulate AS160 phosphorylation in response to insulin, AICAR, and contraction in mouse skeletal muscle. Diabetes 55, 2067-2076. doi: 10.2337/db06-0150

Kuo, C. H., Browning, K. S., and Ivy, J. L. (1999). Regulation of GLUT4 protein expression and glycogen storage after prolonged exercise. Acta Physiol. Scand. 165, 193-201. doi: 10.1046/j.1365-201x.1999.00489.x

Lambeth, M. J., and Kushmerick, M. J. (2002). A computational model for glycogenolysis in skeletal muscle. Ann. Biomed. Eng. 30, 808-827. doi: $10.1114 / 1.1492813$

Leick, L., Fentz, J., Biensø, R. S., Knudsen, J. G., Jeppesen, J., Kiens, B., et al. (2010). PGC-1\{alpha\} is required for AICAR-induced expression of GLUT4 and mitochondrial proteins in mouse skeletal muscle. Am. J. Physiol. Endocrinol. Metab. 299, E456-E465. doi: 10.1152/ajpendo.00648.2009
MacLean, P. S., Zheng, D., and Dohm, G. L. (2000). Muscle glucose transporter (GLUT 4) gene expression during exercise. Exerc. Sport Sci. Rev. 28, 148-152.

Mohsin, A. A., Chen, Q., Quan, N., Rousselle, T., Maceyka, M. W., Samidurai, A., et al. (2019). Mitochondrial complex I inhibition by metformin limits reperfusion injury. J. Pharmacol. Exp. Ther. 369, 282-290. doi: 10.1124/ jpet.118.254300

Narkar, V. A., Downes, M., Yu, R. T., Embler, E., Wang, Y.-X., Banayo, E., et al. (2008). AMPK and PPARdelta agonists are exercise mimetics. Cell 134, 405-415. doi: 10.1016/j.cell.2008.06.051

Olesen, J., Kiilerich, K., and Pilegaard, H. (2010). PGC-1alpha-mediated adaptations in skeletal muscle. Pflugers Arch. 460, 153-162. doi: 10.1007/s00424-010-0834-0

Opie, L. H. (1991). The Heart: Physiology and Metabolism. New York: Raven Press.

Owen, M. R., Doran, E., and Halestrap, A. P. (2000). Evidence that metformin exerts its anti-diabetic effects through inhibition of complex 1 of the mitochondrial respiratory chain. Biochem. J. 348, 607-614. doi: 10.1042/bj3480607

Pederson, B. A., Cope, C. R., Schroeder, J. M., Smith, M. W., Irimia, J. M., Thurberg, B. L., et al. (2005). Exercise capacity of mice genetically lacking muscle glycogen synthase: in mice, muscle glycogen is not essential for exercise. J. Biol. Chem. 280, 17260-17265. doi: 10.1074/jbc.M410448200

Petrosino, J. M., Heiss, V. J., Maurya, S. K., Kalyanasundaram, A., Periasamy, M., LaFountain, R. A., et al. (2016). Graded maximal exercise testing to assess mouse cardio-metabolic phenotypes. PLoS One 11:e0148010. doi: 10.1371/ journal.pone.0148010

Qamar, A., Zhao, J., Xu, L., McLeod, P., Huang, X., Jiang, J., et al. (2021). Cyclophilin D regulates the nuclear translocation of AIF, cardiac endothelial cell necroptosis and murine cardiac transplant injury. Int. J. Mol. Sci. 22:11038. doi: 10.3390/ijms222011038

Radhakrishnan, J., Baetiong, A., Kaufman, H., Huynh, M., Leschinsky, A., Fresquez, A., et al. (2019). Improved exercise capacity in cyclophilin-D knockout mice associated with enhanced oxygen utilization efficiency and augmented glucose uptake via AMPK-TBC1D1 signaling nexus. FASEB J. 33, 11443-11457. doi: 10.1096/fj.201802238R

Radhakrishnan, J., Bazarek, S., Chandran, B., and Gazmuri, R. J. (2015). Cyclophilin-D: A resident regulator of mitochondrial gene expression. FASEB J. 29, 2734-2748. doi: 10.1096/fj.14-263855

Rogatzki, M. J., Ferguson, B. S., Goodwin, M. L., and Gladden, L. B. (2015). Lactate is always the end product of glycolysis. Front. Neurosci. 9:22. doi: 10.3389/fnins.2015.00022

Schinzel, A. C., Takeuchi, O., Huang, Z., Fisher, J. K., Zhou, Z., Rubens, J., et al. (2005). Cyclophilin D is a component of mitochondrial permeability transition and mediates neuronal cell death after focal cerebral ischemia. Proc. Natl. Acad. Sci. U. S. A. 102, 12005-12010. doi: 10.1073/pnas.0505294102

Stanley, W. C., and Chandler, M. P. (2002). Energy metabolism in the normal and failing heart: potential for therapeutic interventions. Heart Fail. Rev. 7, 115-130. doi: 10.1023/A:1015320423577

Tavecchio, M., Lisanti, S., Bennett, M. J., Languino, L. R., and Altieri, D. C. (2015). Deletion of Cyclophilin D impairs $\beta$-oxidation and promotes glucose metabolism. Sci. Rep. 5:15981. doi: 10.1038/srep15981

Tavecchio, M., Lisanti, S., Lam, A., Ghosh, J. C., Martin, N. M., O'Connell, M., et al. (2013). Cyclophilin D extramitochondrial signaling controls cell cycle progression and chemokine-directed cell motility. J. Biol. Chem. 288, 5553-5561. doi: $10.1074 /$ jbc.M112.433045

Taylor, E. B., An, D., Kramer, H. F., Yu, H., Fujii, N. L., Roeckl, K. S. C., et al. (2008). Discovery of TBC1D1 as an insulin-, AICAR-, and contractionstimulated signaling nexus in mouse skeletal muscle. J. Biol. Chem. 283, 9787-9796. doi: 10.1074/jbc.M708839200

Tobias, I. S., Lazauskas, K. K., Siu, J., Costa, P. B., Coburn, J. W., and Galpin, A. J. (2020). Sex and fiber type independently influence AMPK, TBC1D1, and TBC1D4 at rest and during recovery from high-intensity exercise in humans. J. Appl. Physiol. 128, 350-361. doi: 10.1152/japplphysiol.00704.2019

Treebak, J. T., Glund, S., Deshmukh, A., Klein, D. K., Long, Y. C., Jensen, T. E., et al. (2006). AMPK-mediated AS160 phosphorylation in skeletal muscle is dependent on AMPK catalytic and regulatory subunits. Diabetes 55, 2051-2058. doi: $10.2337 / \mathrm{db} 06-0175$

Treebak, J. T., Pehmøller, C., Kristensen, J. M., Kjøbsted, R., Birk, J. B., Schjerling, P., et al. (2014). Acute exercise and physiological insulin induce distinct phosphorylation signatures on TBC1D1 and TBC1D4 proteins in human skeletal muscle. J. Physiol. 592, 351-375. doi: 10.1113/ jphysiol.2013.266338 
Tubbs, E., Theurey, P., Vial, G., Bendridi, N., Bravard, A., Chauvin, M.-A., et al. (2014). Mitochondria-associated endoplasmic reticulum membrane (MAM) integrity is required for insulin signaling and is implicated in hepatic insulin resistance. Diabetes 63, 3279-3294. doi: 10.2337/db13-1751

Vichaiwong, K., Purohit, S., An, D., Toyoda, T., Jessen, N., Hirshman, M. F., et al. (2010). Contraction regulates site-specific phosphorylation of TBC1D1 in skeletal muscle. Biochem. J. 431, 311-320. doi: 10.1042/BJ20101100

Winder, W. W., and Hardie, D. G. (1996). Inactivation of acetyl-CoA carboxylase and activation of AMP-activated protein kinase in muscle during exercise. Am. J. Phys. 270, E299-E304. doi: 10.1152/ajpendo.1996.270.2.E299

Woods, A., Johnstone, S. R., Dickerson, K., Leiper, F. C., Fryer, L. G. D., Neumann, D., et al. (2003). LKB1 is the upstream kinase in the AMPactivated protein kinase cascade. Curr. Biol. 13, 2004-2008. doi: 10.1016/j. cub.2003.10.031

Zhou, G., Myers, R., Li, Y., Chen, Y., Shen, X., Fenyk-Melody, J., et al. (2001). Role of AMP-activated protein kinase in mechanism of metformin action. J. Clin. Invest. 108, 1167-1174. doi: 10.1172/JCI13505
Conflict of Interest: The authors declare that the research was conducted in the absence of any commercial or financial relationships that could be construed as a potential conflict of interest.

Publisher's Note: All claims expressed in this article are solely those of the authors and do not necessarily represent those of their affiliated organizations, or those of the publisher, the editors and the reviewers. Any product that may be evaluated in this article, or claim that may be made by its manufacturer, is not guaranteed or endorsed by the publisher.

Copyright (๑ 2021 Radhakrishnan, Baetiong and Gazmuri. This is an open-access article distributed under the terms of the Creative Commons Attribution License (CC BY). The use, distribution or reproduction in other forums is permitted, provided the original author(s) and the copyright owner(s) are credited and that the original publication in this journal is cited, in accordance with accepted academic practice. No use, distribution or reproduction is permitted which does not comply with these terms. 